\title{
Analysis of mode coupling due to spherical defects in ideal fully metal-coated scanning near-field optical microscopy probes
}

\author{
Wataru Nakagawa, Luciana Vaccaro, and Hans Peter Herzig \\ Institute of Microtechnology, University of Neuchâtel, Rue A.-L. Breguet 2, 2000 Neuchâtel, Switzerland
}

\begin{abstract}
Received July 19, 2005; revised October 12, 2005; accepted November 2, 2005; posted November 9, 2005 (Doc. ID 63526)
We investigate the effect of defects in the metal-coating layer of a scanning near-field optical microscopy (SNOM) probe on the coupling of polarization modes using rigorous electromagnetic modeling tools. Because of practical limitations, we study an ensemble of simple defects to identify important trends and then extrapolate these results to more realistic structures. We find that a probe with many random defects will produce a small but significant coupling of energy between a linearly polarized input mode and a radial/longitudinal polarization mode, which is known to produce a strongly localized emitted optical field and is desirable for SNOM applications. () 2006 Optical Society of America

OCIS codes: $180.5810,260.5430,350.5500$.
\end{abstract}

\section{INTRODUCTION}

Recently, a significant amount of interest has been given to fully metal-coated scanning near-field optical microscopy (SNOM) probes. ${ }^{1,2}$ While the total light throughput of these probes is generally lower than for uncoated or partially metal-coated tapered-fiber probes, they provide other advantages, such as high-volume manufacturability with standard fabrication techniques and materials, as well as greater manufacturing reproducibility and control over the device shape. These probes also possess a significant advantage in that optical as well as topographic (e.g., atomic force microscope) measurements can be performed using the same tip. ${ }^{2}$ To better understand the operating characteristics of this class of probes, a considerable amount of work has been performed to optimize their fabrication, as well as to experimentally measure their performance characteristics. ${ }^{3-8}$ In particular, the properties of the metal coating ${ }^{9,10}$ and the polarization state of the light transiting the probe ${ }^{11-13}$ have been found to play an important role in the optical properties and performance capabilities of the probe. However, as fabricating and testing large numbers of probes with varying configurations is presently impractical, rigorous electromagnetic modeling of such probes has proven to be a useful complement to experimental work in gaining insight into the propagation of light inside and near such probes.

In previous numerical studies of apertureless probes, it was shown that the emitted near field at the apex of the probe tip depends strongly on the polarization state of the input field. The lowest-order eigenmodes of the probe input aperture are a pair of orthogonal linearly polarized modes, followed by a mode with a dominant radial or longitudinal polarization configuration. While the linearly polarized modes were found to transmit more power through the probe, the radial/longitudinal polarization mode was found to produce a highly localized field hot spot in the near-field zone of the probe apex. ${ }^{11}$ In addition to being confined to a smaller volume, the peak field am- plitude of this polarization mode is significantly higher than for the linearly polarized modes. Subsequent experimental studies confirmed these numerical results, finding that injecting the radial/longitudinal polarization mode into a microfabricated probe does in fact produce a more strongly localized emitted optical near field, as compared with injecting a purely transversely polarized mode. ${ }^{12,13}$ For high-resolution microscopy applications, this strong localization of the emitted field is clearly desirable. The remaining challenge is to simplify the creation and injection of such a radial/longitudinal polarization mode. One promising approach is to modify the probe structure in some way to produce a conversion mechanism from a more straightforward linearly polarized input mode to the radial/longitudinal mode emitting a highly confined optical near field.

In this work we investigate the role of various types of defects in the metal-coating layer of the probe on the emitted optical fields, including coupling between the key polarization modes. We focus on defects in the metal layer as they most closely correspond to the observed characteristics of real manufactured probes. ${ }^{2,6}$ Because of restrictions based on an unambiguous interpretation of results as well as computational load limitations, we focus on studying an ensemble of simple but insightful defect configurations in a simplified model of the probe. Although these simplified models do not accurately reflect the characteristics of real probes in their entirety, they do facilitate understanding the fundamental mechanisms of optical coupling between the polarization modes in the probe. Furthermore, comparisons between the various defect configurations studied, including defect position, size, and shape, as well as multiple defects, do provide meaningful insight into the polarization-dependent characteristics of this type of structure. These results can also be extrapolated to predict the likely aggregate optical behavior of a large number of random defects - corresponding to the polarization characteristics of realistic probes. 


\section{BACKGROUND}

\section{A. Modeling Approach}

The study of the interaction of optical fields with physical structures having nanoscale features requires an analysis technique that is both highly accurate and flexible enough to handle a variety of material and field configurations. To fulfill these requirements, we choose the Microwave Studio software package from Computer Simulation Technologies, in Darmstadt, Germany. This tool is based on the finite-integration time-domain technique and provides the required accuracy and flexibility to investigate this class of structures.

A schematic diagram of the simplified SNOM probe model is shown in Fig. 1. The overall modeling domain is $1 \mu \mathrm{m} \times 1 \mu \mathrm{m} \times 1.7 \mu \mathrm{m}$ in the $x, y$, and $z$ directions, respectively. The probe axis is chosen to lie along the $z$ axis. The probe itself consists of a silica core $(n=1.5)$ and an alumi-

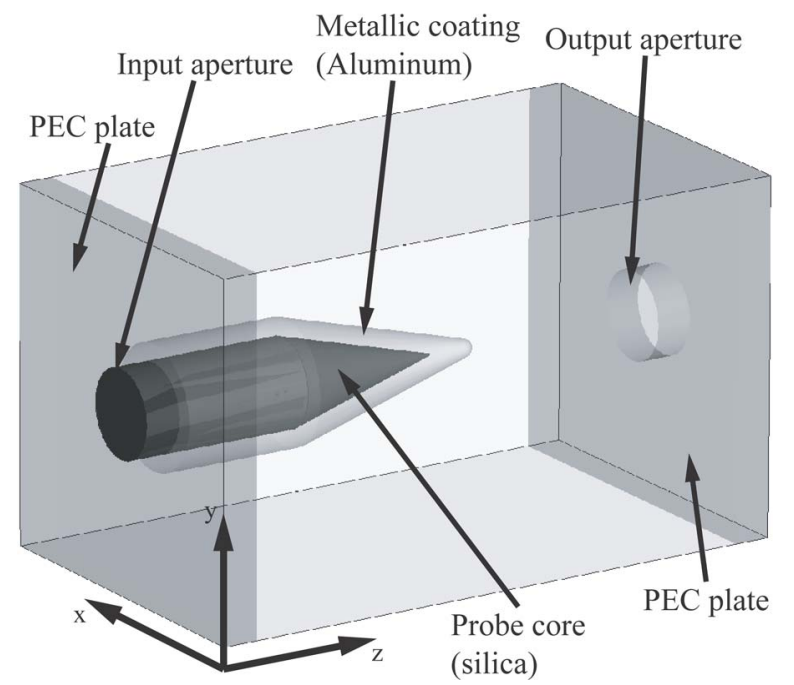

Fig. 1. Diagram of the three-dimensional modeling domain. The tip of the probe is modeled as a cylindrical silica core with a conical taper having a $30^{\circ}$ apex angle and an approximately $50 \mathrm{~nm}$ thick aluminum coating. A $0.1 \mu \mathrm{m}$ thick perfect electric conductor (PEC) plate is placed at each end of the domain along the $z$ axis, with a $250 \mathrm{~nm}$ diameter hole in each forming the input and output apertures. The lateral ( $x$ and $y$ ) edges of the domain utilize an absorbing boundary condition. num coating (dispersive, with $n=0.645+5.029 i$ at the operating wavelength $\lambda=532 \mathrm{~nm}$ ). The core is composed of a cylindrical section $250 \mathrm{~nm}$ in diameter and $0.5 \mu \mathrm{m}$ high, followed by a conical taper $467 \mathrm{~nm}$ high. This yields an apex angle of $30^{\circ}$, consistent with experimental studies currently being conducted. ${ }^{12,13}$ The metal coating is a uniform $50 \mathrm{~nm}$ thick layer of aluminum on both the cylindrical and the tapered sections, terminating in a spherical apex of variable diameter (typically $50 \mathrm{~nm}$ ). Before the subsequent introduction of defects, the probe exhibits a rotational symmetry around the $z$ axis.

A $0.1 \mu \mathrm{m}$ thick layer of a perfect electric conductor (PEC) is placed at each end of the domain along the $z$ axis. A circular hole of diameter $250 \mathrm{~nm}$ is embedded in each of these PEC layers, forming the input and output apertures. At the base of the probe (the input aperture), the aperture is composed of silica to match the cylindrical section of the probe. At the opposite end of the domain (the output aperture), the aperture consists of air to match the region surrounding the probe. Although the presence of the PECs certainly perturbs the distribution of the optical fields to some extent, this is not likely to be critical in a comparative analysis of various probe configurations, where the matched input and output ports provide a simple and useful mechanism for evaluating the polarization mode coupling. An absorbing boundary condition is applied on the lateral $(x$ and $y$ ) edges of the domain.

\section{B. Fundamental Polarization Modes}

For the input aperture of the model, we can compute the eigenmodes of this input port. Although numerous modes exist, the first three are of the greatest interest in this work, and are shown in Fig. 2. First, shown in Fig. 2(a) is a primarily linearly polarized mode with a dominant $E$-field vector parallel to the $x$ axis. This mode is called $\mathrm{H}$ for simplicity in the subsequent discussion. Next is the orthogonal linearly polarized mode with a dominant $E$-field vector parallel to the $y$ axis, shown in Fig. 2(b) and called $\mathrm{V}$ for simplicity. Finally, there is the radially polarized or longitudinally polarized mode as shown in Fig. 2(c), called R. From previous studies, this mode is responsible for pro-

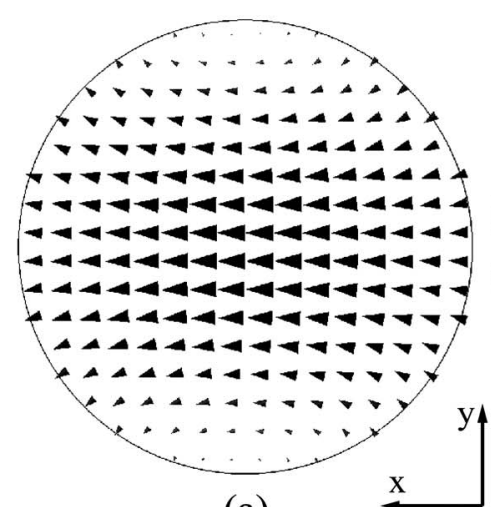

(a)

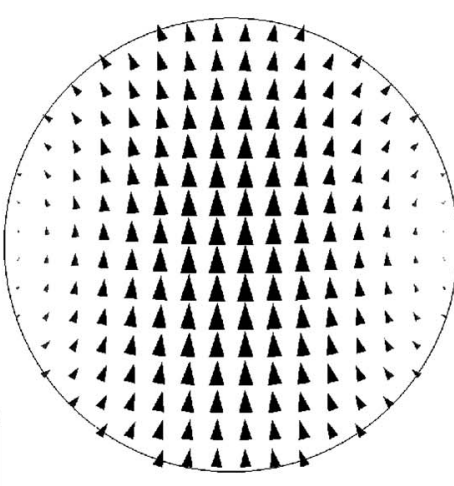

(b)

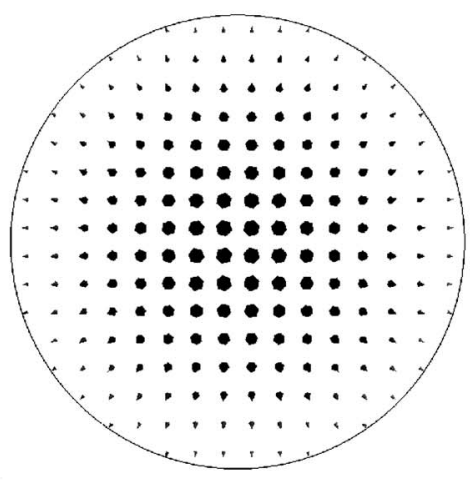

(c)

Fig. 2. Images of the three fundamental polarization eigenmodes for the input and output apertures of the model: (a) horizontal linearly polarized mode $\mathrm{H}$ with a dominant electric field component along the $x$ axis; (b) vertical linearly polarized mode $\mathrm{V}$ with a dominant electric field component along the $y$ axis; (c) radial/longitudinal polarized mode $\mathrm{R}$ with a dominant electric field component along the $z$ axis. 


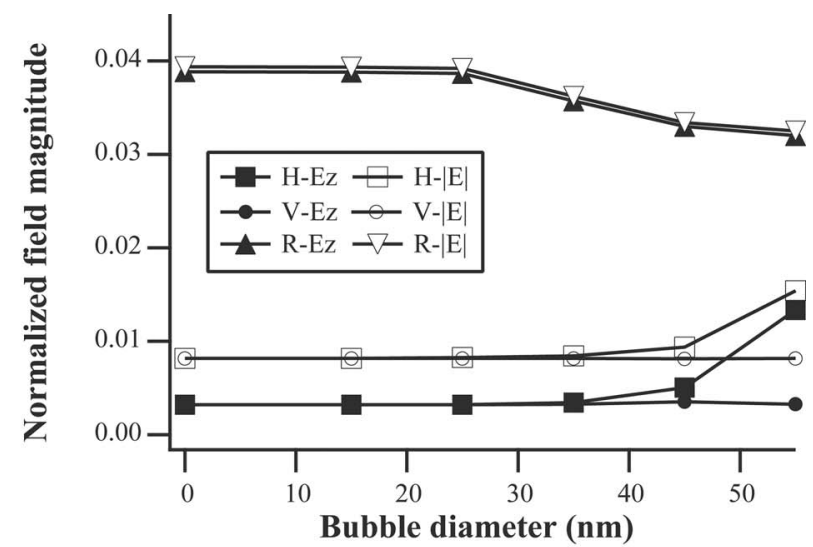

(a)

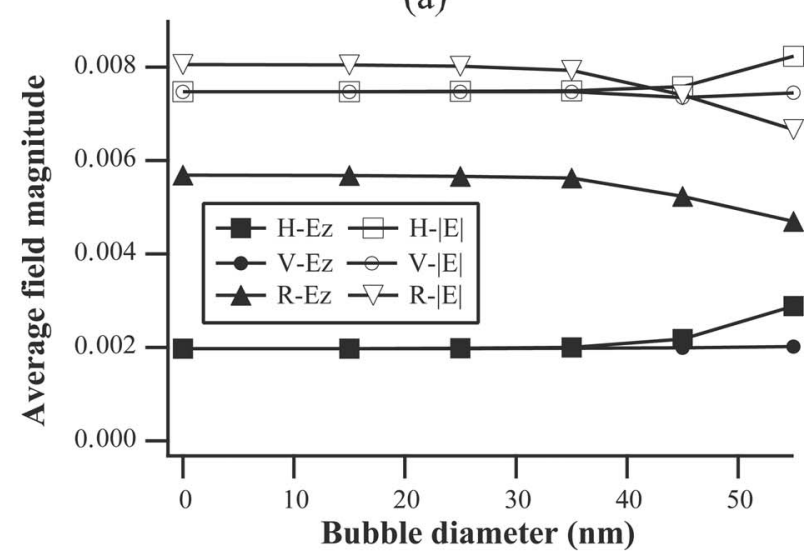

(b)

Fig. 3. (a) Normalized peak field magnitude at an observation point on the probe axis $10 \mathrm{~nm}$ beyond the apex as a function of spherical bubble defect diameter. (b) Normalized average field magnitude over a region $120 \mathrm{~nm} \times 120 \mathrm{~mm}$ centered on the probe axis located $10 \mathrm{~nm}$ beyond the probe apex as a function of bubble diameter. The normalization is performed with respect to the peak magnitude of the input mode at the input aperture.

ducing a highly localized optical field hot spot in the nearfield zone of the probe. ${ }^{11}$

In the following investigation, we take the incident optical field at the input port to be one of these three eigenmodes: $\mathrm{H}, \mathrm{V}$, or $\mathrm{R}$. The analysis of the output of the probe centers on one of two cases: (1) an evaluation of the emitted field near the apex of the probe or (2) the coupling efficiency to the same three modes $\mathrm{H}, \mathrm{V}$, and $\mathrm{R}$ at the output port. Although coupling to higher-order modes does exist, it is generally significantly weaker than for the three modes under consideration, and is ignored in this analysis.

\section{SINGLE SPHERICAL DEFECT}

To investigate the influence of defects on the propagation characteristics of the fundamental optical polarization modes in the probe model, we introduce a simple modification or defect into the structure: the inclusion of a spherical air bubble in the metal-coating layer of the probe. In the first study, the bubble consists of air ( $n$ $=1.0$ ) and is located in the center of the metal layer, at the midpoint of the tapered section of the probe. We vary the size of the bubble and observe the effect on the transmis- sion characteristics of the probe. The size varies from a diameter of $15-55 \mathrm{~nm}$ in steps of $10 \mathrm{~nm}$. With a metal layer thickness of $50 \mathrm{~nm}$, the largest bubble just breaks the surface of the metal on both sides. In this case the bubble does not penetrate the silica underneath the metal, instead leaving a small area of silica exposed to the air. For simplicity, we position the bubble in the $X-Z$ plane, such that it lies in the plane parallel to the incident $E$-field vector for the input mode $\mathrm{H}$.

\section{A. Near-field Amplitude}

To observe the effect of the bubble on the emitted optical near field of the probe, we first examine the field amplitude at a point located $10 \mathrm{~nm}$ away from the apex along the probe axis. This approximates the closest approach of an object being scanned by the probe under normal operating conditions. The results for the total field amplitude as well as the amplitude of the $z$ component are shown only as a function of the bubble diameter in Fig. 3(a). The case without a defect is denoted in the figures as bubble diameter zero. The field magnitude is normalized against the peak amplitude of the input mode at the input aperture, giving a sense of the optical coupling through the probe. First, note that for small bubble diameters, the emitted field for the two transverse modes $\mathrm{H}$ and $\mathrm{V}$ are essentially identical, as there is a rotational symmetry in the unperturbed probe. Also, we observe that the field amplitude for the mode $\mathrm{R}$ is significantly higher than for the $\mathrm{H}$ and $\mathrm{V}$ modes. This result is consistent with previous investigations ${ }^{11}$ predicting a highly localized optical field in the immediate vicinity of the probe apex for input mode R. Next, as the bubble diameter approaches the larger values $(35,45,55 \mathrm{~nm})$, there is a clear change in the near field emitted by the probe except for the mode V, which exhibits essentially constant behavior for all bubble diameters. For the transverse mode $\mathrm{H}$, we see that the observed field amplitude increases as the bubble diameter increases. In addition, the $z$ component of the field becomes an increasingly large fraction of the total, indicating that the $z$ component of the field is becoming dominant. This suggests that even with the $\mathrm{H}$ input mode, due to the presence of the bubble defect in the metal layer, a field hot spot as seen for the mode $\mathrm{R}$ is developing in the near-field zone of the probe. Conversely, looking at the emitted near field for mode $\mathrm{R}$, we see that there is a slight decrease in the observed field amplitude as the bubble diameter increases. Taken together, these two observations suggest that there is a coupling induced between the modes $\mathrm{H}$ and $\mathrm{R}$ due to the presence of the bubble. Nevertheless, even for the largest bubble diameter considered, the maximum field amplitude observed for the $\mathrm{H}$ and $\mathrm{V}$ modes is significantly lower than for the mode R. This indicates that while this mode coupling may be significant, it is never dominant, at least with this probe configuration.

It is also useful to look at the average field amplitude (again, total and $z$ component only, normalized with respect to the peak amplitude of the input mode) over a small region in the $x-y$ plane $(120 \mathrm{~nm} \times 120 \mathrm{~mm})$ located $10 \mathrm{~nm}$ away from the probe apex. These results as a function of the bubble diameter are shown in Fig. 3(b). For input mode R, while the average of the $z$ component of the 
field over this small region is still significantly higher than for the linearly polarized modes, the $z$ component of the field is a much smaller fraction of the total field, indicating that the transverse ( $x$ and $y$ ) components of the field are also significant off axis. Also, the average of the total field magnitude over this small region is approximately the same for all three polarization modes. Coupled with the previous results, this result is consistent with mode $\mathrm{R}$ producing a strongly localized field with a high peak amplitude in the near-field zone of the probe, while the linearly polarized modes $\mathrm{H}$ and $\mathrm{V}$ produce a less strongly confined emitted field. Finally, while the mode V is largely unaffected by the presence of the defect, increasing the size of the bubble in the metal layer results in a reduced average emitted field for mode $R$ and an increased average emitted field for mode $\mathrm{H}$. This is consistent with the previous results, and again suggests that the presence of this spherical air defect introduces a coupling between modes $\mathrm{H}$ and $\mathrm{R}$ in the probe structure.

\section{B. Mode-Coupling Coefficients}

Although the amplitude of the optical field in the nearfield zone of the probe is the most important characteristic of interest in this analysis of near-field probe behavior, it is not necessarily the most convenient method for comparing the characteristics of different probes. While the results of the previous subsection suggest that the defect induces a coupling between two of the polarization eigenmodes, interpretation of these results is complicated by the fact that the actual optical field at the point of observation is the superposition of at least two probe polarization modes. Thus, in order to more clearly observe the coupling between these modes, instead of studying the emitted near field, we consider the mode-to-mode coupling efficiencies between the input and output ports as shown in Fig. 1. In this investigation, the model including the probe structure remains exactly the same as in the previous example, only the optical parameters under observation are different.

The output port has the same dimensions as the input port, and the three polarization eigenmodes under consideration are qualitatively identical at the two ports. The coupling coefficient for a given mode pair is the squared magnitude of the ratio of the output and input mode am-

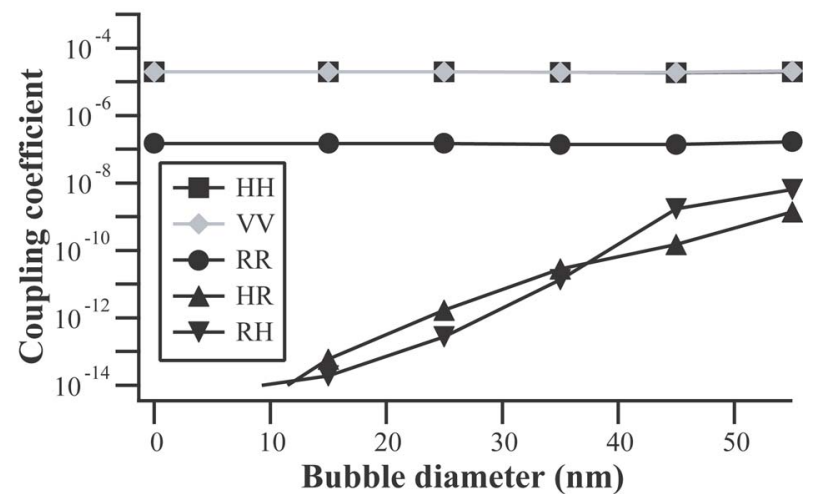

Fig. 4. Input-to-output port mode-coupling coefficients as a function of defect diameter for a single air bubble placed in the metal-coating layer at the midpoint of the tapered section in the $x-z$ plane. plitudes (in rf/waveguide notation: $\left|S_{21}\right|^{2}$ ). The nine possible coupling coefficients between the modes $H, V$, and $R$ at the input and output ports as a function of the bubble diameter are shown in Fig. 4. Once again, the case of having no defect is represented as a bubble diameter of zero. The coefficients, reflecting the coupling of energy from a given input polarization mode to a given output mode, are shown on a logarithmic amplitude scale. First, we note that the coupling between the like transverse modes, $\mathrm{HH}$ (i.e., input mode $\mathrm{H}$ coupling to output mode $\mathrm{H}$ ) and VV, are equal, roughly constant with respect to the bubble diameter, and the largest of all in magnitude. Next, the radial mode-coupling coefficient $R R$ is also constant, although it is approximately two orders of magnitude smaller than for the linearly polarized modes. This is in contrast to the previous study, where the input mode $R$ was found to produce an emitted optical near field significantly stronger than that of the modes $\mathrm{H}$ and $\mathrm{V}$. This outcome is due to two principal factors: First, while the emitted field for the mode $\mathrm{R}$ has a much higher peak amplitude due to the strong localization, the total amount of power emitted is lower. Second, the strongly localized field hot spot for the mode $\mathrm{R}$ will diffract very strongly before propagating even the short distance to the output port. Most importantly, in Fig. 4 the only coupling coefficients that show a clear dependence on the bubble diameter are between the modes $\mathrm{H}$ and $\mathrm{R}$. For both $\mathrm{HR}$ and $\mathrm{RH}$, the observed coupling uniformly increases as the bubble diameter is increased. These results confirm the previous hypothesis that the defect introduces a coupling between these two modes. Finally, the remaining crosscoupling coefficients, between modes $\mathrm{V}$ and $\mathrm{R}$ as well as $\mathrm{H}$ and $\mathrm{V}$, remain negligibly small and are omitted from the figure.

\section{Discussion}

In this section we have presented two parallel investigations of the same set of probe models-containing a single spherical air bubble defect of varying diameter in the center of the metal-coating layer-focusing on different optical output characteristics. In the first case, we find that even with a linearly polarized input mode $(\mathrm{H})$, due to the presence of the bubble, a small but nonnegligible highly localized emitted optical field (very similar to that expected for the radially polarized input mode $R$ ) is observed in the near-field zone of the probe. In the second case, considering the mode-to-mode coupling coefficients between the input and output ports of the model, we find that the only clear correlation with the bubble diameter is for coupling between modes $\mathrm{H}$ and $\mathrm{R}$. Clearly, there is a strong connection between the mode coupling HR and the emergence of the near-field hot spot in the emitted optical field. While we have not conclusively demonstrated a quantitative link between these two phenomena, the strong qualitative relationship established in this section enables characterization of certain near-field optical properties of various probe models by analyzing the polarization mode coupling coefficients.

Ultimately, the emitted optical field in the near-field zone of the probe is the characteristic of interest in this investigation. However, as mentioned previously, once nontrivial defects or other perturbations are introduced 
into the probe structure, the emitted optical field becomes a superposition of modes. Consequently, it becomes difficult to find a simple and insightful means to represent the effect of various defects based on the properties of the emitted field. For example, there are many potentially meaningful characteristics-field amplitude at a particular point of interest, peak field amplitude, average field over a given volume, size of the emitted field hot spot, or ratio of the hot spot peak to the background amplitude, among others-making it difficult to choose an appropriate metric to describe and compare the effects of various defect configurations. Fortunately, we know that the desirable highly localized near-field hot spot is produced by the radial/azimuthal polarization mode $\mathrm{R} .{ }^{11}$ This fact, together with the results of this section, suggests that the coupling of the modes $\mathrm{H}$ and $\mathrm{V}$ with the mode $\mathrm{R}$ in the probe, rather than the emitted field characteristics, best describes the phenomena of interest. Thus, in general, the polarization mode coupling coefficients provide a simple and meaningful framework for representing and, more important, comparing the effects of interest for various defect configurations.

\section{MULTIPLE DEFECTS}

Using the approach described in the previous sectioncomparing the input mode to output mode coupling coefficients-we now investigate several additional defect configurations. Ideally, in order to directly relate these results to experimental work using this type of probe, we would like to investigate a real probe containing a large number of random defects (see, for example, Fig. 2 in Ref. 6 and Fig. 8 in Ref. 13). Unfortunately, at present such a model is impractical due to limitations in computational power and the difficulty of interpreting and analyzing the results produced by such a complex structure. Instead, as in the previous section, we consider several series of related simple examples. While each of these cases consists of an ideal probe model with one or a few specific introduced defects, the ensemble of cases is chosen to illustrate several important general trends that can be extrapolated to draw some qualitative conclusions about real, random probes. In the following, we consider three such ensembles of defect configuration in the probe model: (1) the longitudinal placement of one or more spherical air defects, (2) the axial placement of pairs of spherical air defects, and (3) several examples of more realistic-but still very simple-defects.

\section{A. Defect Longitudinal Placement}

In the first set of examples, we consider the placement of a spherical air bubble defect in the probe. Several cases are considered, as shown in Fig. 5(a). The index numbers 1-7 refer to the bubble positions as shown, all in the $x-z$ plane. As the results of Fig. 5(b) show, the only clear dependence of the mode coupling due to the position of the bubble is between modes $\mathrm{H}$ and $\mathrm{R}$. The coupling between modes $\mathrm{H}$ and $\mathrm{V}$ as well as modes $\mathrm{V}$ and $\mathrm{R}$ are of a small enough magnitude to be insignificant, and are not shown. Also, the coupling coefficients between like modes $\mathrm{HH}$, $\mathrm{VV}$, and $\mathrm{RR}$ are stronger and are independent of the bubble position. These results are consistent with the pre-

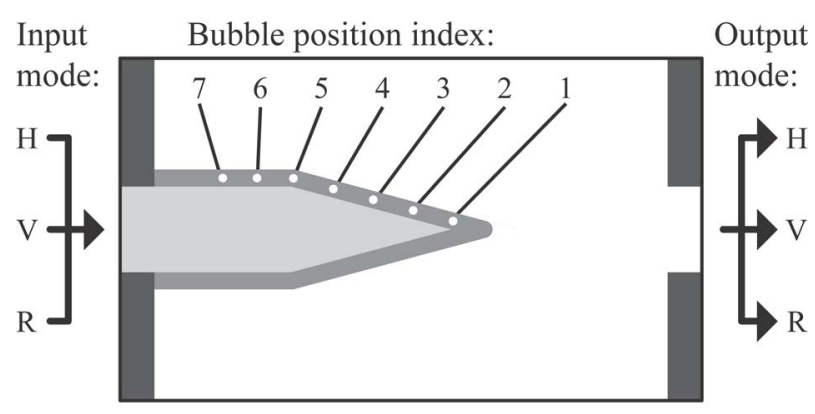

(a)

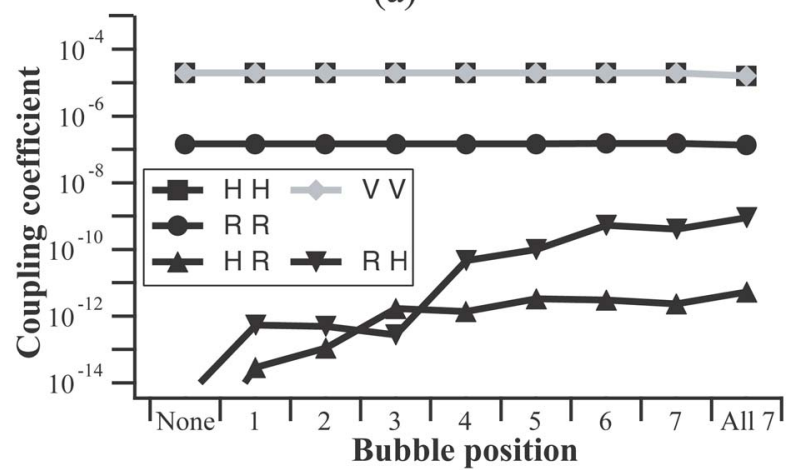

(b)

Fig. 5. (a) Diagram indicating the placement of seven different air-bubble defects in the $x-z$ plane. (b) Input-to-output port mode coupling coefficients for the seven defect configurations shown in (a).

vious examples, and once again show that the inclusion of a bubble in the $x-z$ plane of the probe induces a coupling between modes $\mathrm{H}$ and R. Furthermore, comparing the effects of the various bubble positions, we find that the induced coupling between these two modes is strongest for the bubbles located in the cylindrical section of the probe, with a noticeable decrease in the coupling efficiency for the cases of bubbles located near the probe apex. This is explained by the fact that the field amplitude found inside the probe (including in the metal layer) is significantly higher near the input aperture rather than near the apex, resulting in a stronger net coupling for the highernumbered bubble positions. In addition, we note that the coupling coefficient $\mathrm{RH}$ is generally significantly higher than for HR. In the previous section, a similar effect was found for the coupling of modes $\mathrm{HH}$ and $\mathrm{RR}$ in a probe without a defect. This result is most likely also explained by these differing emitted field characteristics for modes $\mathrm{H}$ and R: less total power emission for mode $\mathrm{R}$ from the probe and stronger localization in the near field resulting in stronger diffraction before reaching the output port.

In addition to the various single-defect configurations, we also consider the case where all seven bubbles are included, as shown in Fig. 5(b). As in all the previous cases, the coupling efficiencies of the three primary modes to like modes are essentially constant. The coupling between modes $\mathrm{H}$ and $\mathrm{R}$ is stronger than for any one of the singlebubble cases, although it is less than the sum of the coupling efficiencies for the seven single-bubble cases. And as before, the cross coupling between other modes is negligible. From this result, we see that in the case where multiple bubbles are present, but confined to a single plane, 
although the absolute strength of the coupling is increased, the qualitative behavior of inducing a coupling between modes $\mathrm{H}$ and $\mathrm{R}$ is not fundamentally affected. Thus, in this configuration, the collective effect of the seven bubbles can be considered to be equivalent to the effect of a single, larger effective bubble.

\section{B. Defect Axial Placement}

Next, we consider probes having two spherical defects. In this set of examples, the azimuthal and longitudinal placement of the two bubbles and their relative sizes play a critical role in determining the net output of the probe. The two-bubble configurations to be studied are shown in Fig. 6, along with three previous cases (no defect, a single 25 or $45 \mathrm{~nm}$ bubble) for comparison.

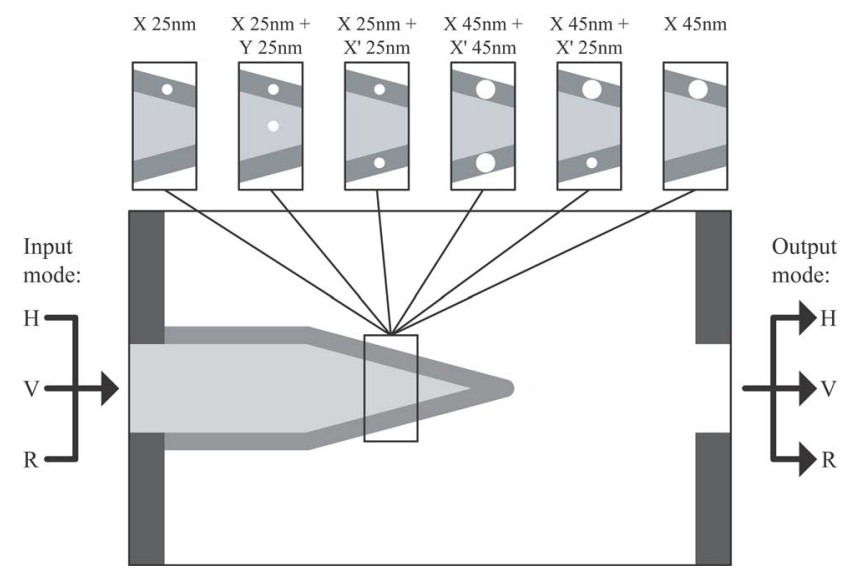

Fig. 6. Diagram indicating the bubble placement for several configurations consisting of one or two spherical air defects in the axial $(x-y)$ plane at the midpoint of the probe tapered section.
The first example is the inclusion of two identical bubbles at equivalent positions in two orthogonal longitudinal planes of the probe. In the previous section, we found that a bubble in plane $x-z$ induces a coupling between modes $\mathrm{H}$ and $\mathrm{R}$. So we expect that the introduction of an identical bubble in the $y-z$ plane will introduce an equivalent coupling between modes $\mathrm{V}$ and $\mathrm{R}$. As shown in Fig. 6, both bubbles have a diameter of $25 \mathrm{~nm}$ and are placed at the midpoint of the tapered section of the probe, one lying in the $x-z$ plane (called bubble $\mathrm{X}$ ) and the other in the $y-z$ plane (called bubble Y). Looking at the results, shown in Fig. 7, we see that this is in fact the case. Each mode is coupled to itself as in the previous case, the two linearly polarized modes $\mathrm{H}$ and $\mathrm{V}$ are each coupled to $\mathrm{R}$, and the coupling between modes $\mathrm{H}$ and $\mathrm{V}$ remains insignificant.

Next, we also consider the case where the two bubbles are placed on opposite sides of the probe. This configuration, also shown in Fig. 6, consists of identical $25 \mathrm{~nm}$ bubbles $\mathrm{X}$ and $\mathrm{X}^{\prime}$ placed at diametrically opposite locations at the middle of the tapered section in the $x-z$ plane of the probe. The coupling coefficient results for this case show essentially identical results for the case of no bubble. This result can be qualitatively explained by considering the fact that the pair of diametrically opposed bubbles will produce identical scattered fields, but with opposite sign in the $z$ components due to the symmetry of the configuration. As the $z$ component of the electric field is critical for mode $R$, we expect the effective destructive interference of the scattered fields due to the two bubbles to result in a net zero coupling to the radial mode. We also consider a similar configuration, with two $45 \mathrm{~nm}$ diametrically opposed bubbles, also shown in Fig. 6 . As in the $25 \mathrm{~nm}$ example, the observed coupling between the modes

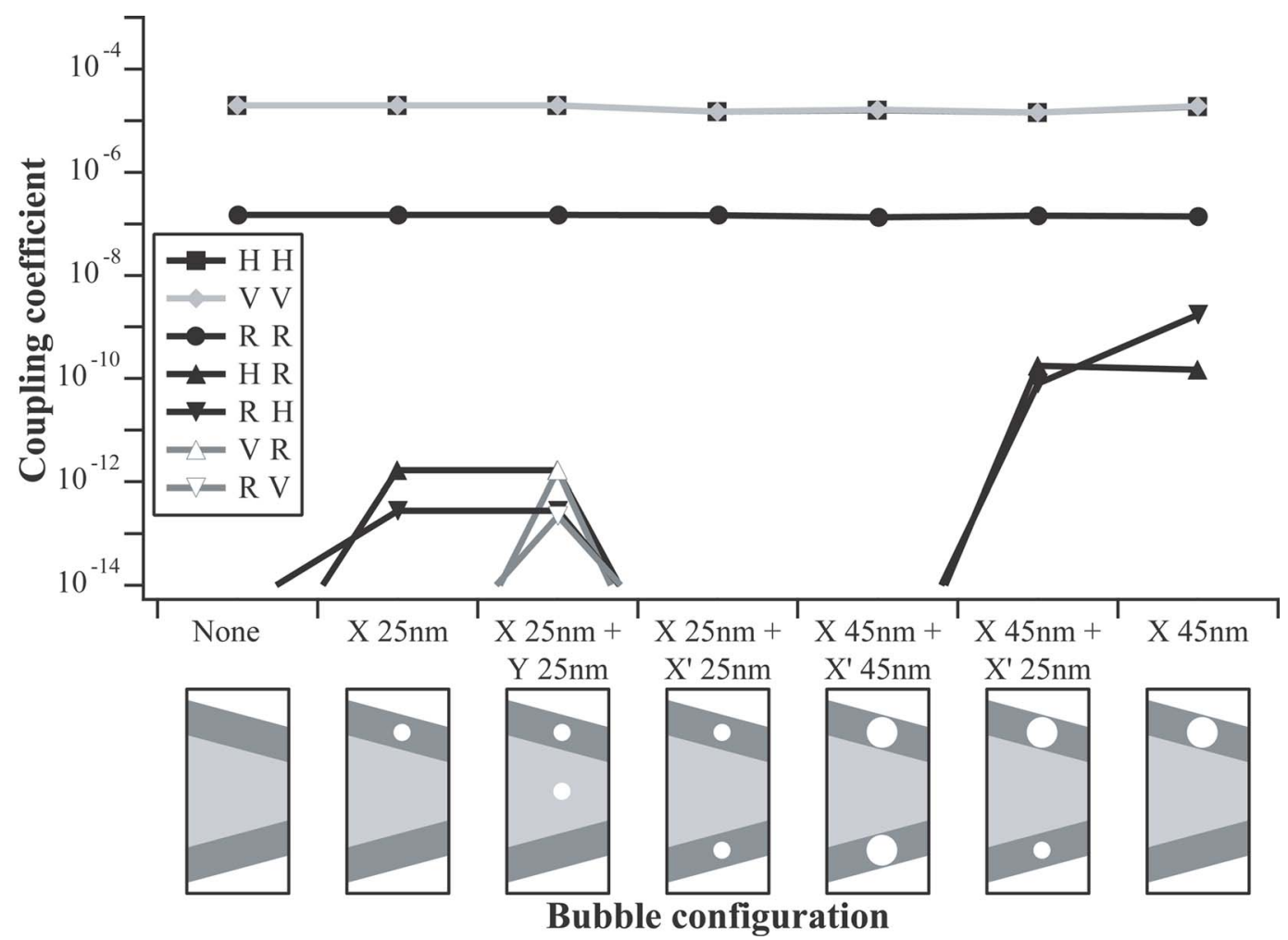

Fig. 7. Input-to-output port mode coupling coefficients for the defect configurations shown in Fig. 6. 
is essentially identical to the case of having no bubble in the probe, due to the destructive interference of the scattering from the two bubbles.

Finally, we also consider the case of the two diametrically opposite bubbles with differing sizes. As shown in Fig. 6, in this case bubble $X$ has a diameter of $45 \mathrm{~nm}$, while bubble $\mathrm{X}^{\prime}$ has a diameter of $25 \mathrm{~nm}$. From the results shown in Fig. 7, the coupling effect of the asymmetric pair of bubbles between modes $H$ and $R$ is stronger than for the single $25 \mathrm{~nm}$ bubble. In comparison with the single $45 \mathrm{~nm}$ bubble, the HR coupling is approximately the same, while the $\mathrm{RH}$ coupling is slightly weaker with the asymmetric pair of bubbles.

Overall, these examples give some further insight into the cumulative behavior of multiple defects. For some cases, such as two bubbles on orthogonal planes, the net effect is exactly the sum of the effects of the individual bubbles. In other specific cases, such as diametrically opposed identical bubbles, destructive interference is observed. However, in general, as these two-bubble examples, as well as the seven-bubble case from the previous section, suggest, the cumulative effect of a number of randomly placed bubbles is likely to be additive, although most likely not in a purely linear way. In other words, in terms of induced coupling between probe polarization eigenmodes, the aggregate effect of a number of bubbles is qualitatively similar to that of a single similar bubble, but with most likely an increased coupling strength. To examine this idea more closely, we consider several examples of more realistic defects, composed of a small number of spherical inclusions or other basic defect shapes.

\section{More Realistic Defects}

Finally, in addition to studying the interaction of specific pairs of bubbles, we also consider several cases of more re- alistic but simple defects, in order to gain insight into the general behavior of multiple, random defects. In the following results, two previous cases, no defect and a single $45 \mathrm{~nm}$ spherical defect, are also shown for comparison purposes.

The first example we consider is a chain of bubbles that forms a tunnel through the metal layer of the probe. Pictures of such a structure, with differing placement in the probe, are shown in the insets of Fig. 8. The defect itself consists of three spheres, with diameters of 25,20 , and $25 \mathrm{~nm}$, placed along an axis perpendicular to the metal surface. The center of the central sphere is at the midpoint of the metal layer, and the two side spheres are each located approximately $8 \mathrm{~nm}$ from the adjacent surface, resulting in a few-nanometer overlap between adjacent spheres. The spheres do not project beyond the boundaries of the metal layer, leaving a small exposed surface of each side sphere flush with the metal surface. For a more

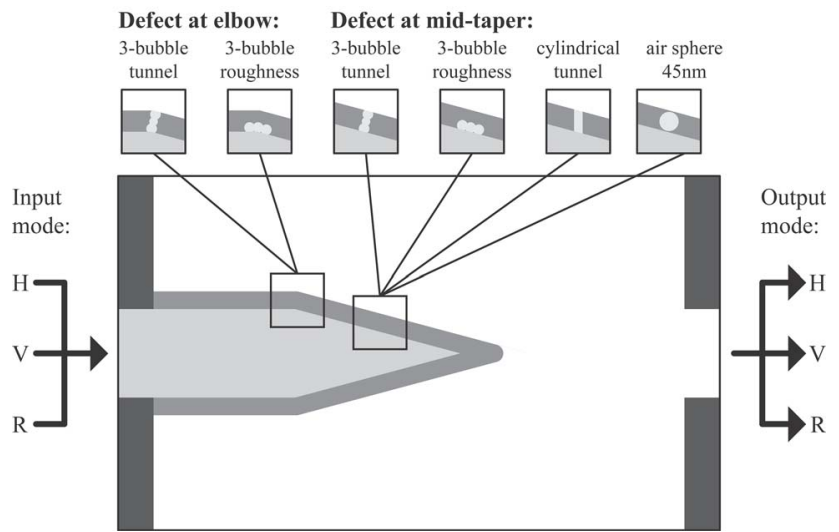

Fig. 8. Diagram showing several examples of simple but more realistic oxidized aluminum $(n=1.54)$ defect configurations.

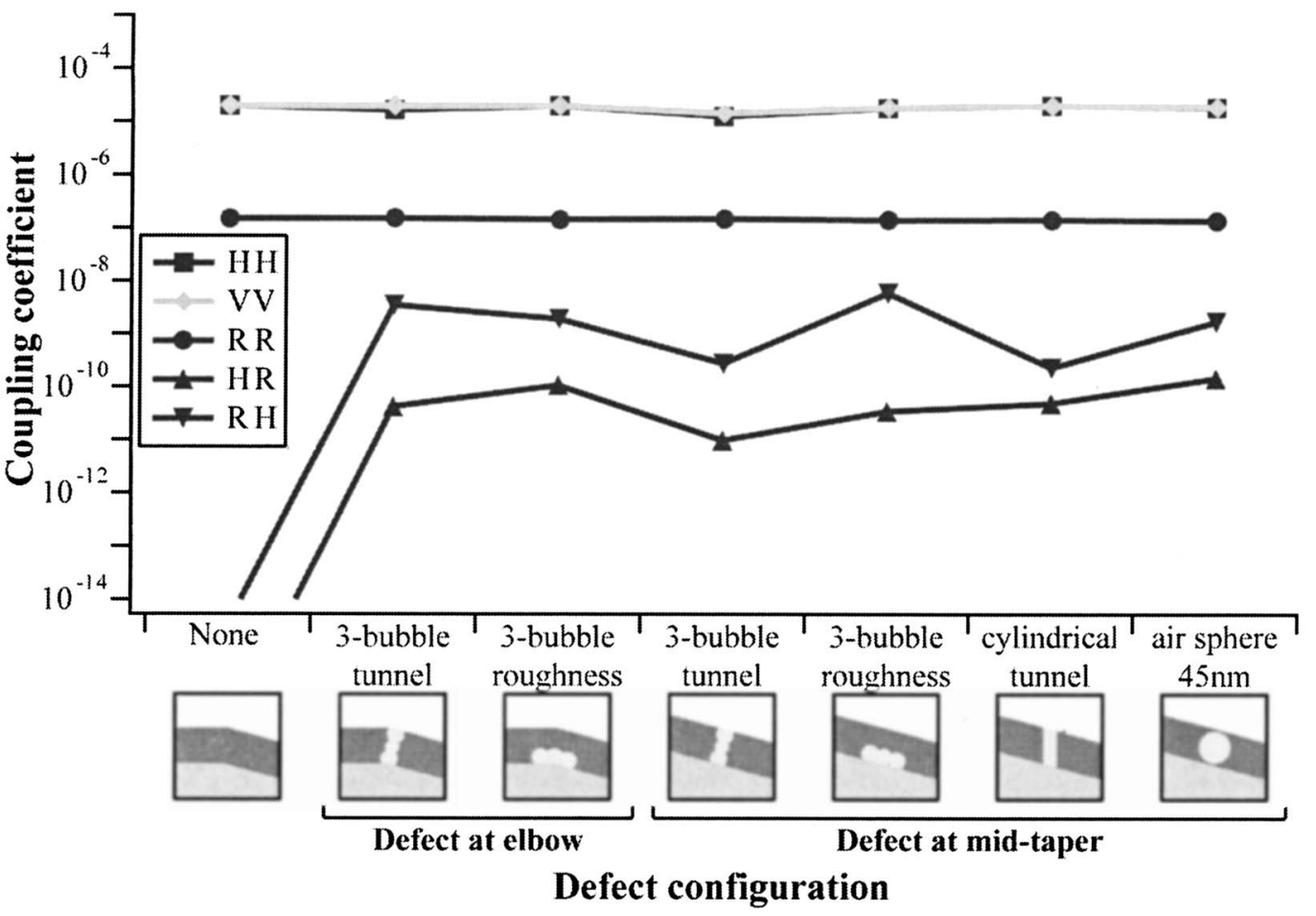

Fig. 9. Input-to-output port mode coupling coefficients for the defect configurations shown in Fig. 8. 
realistic model, the spheres are assumed to be made of oxidized aluminum instead of air, and to have a higher index of refraction $(n=1.54)$. The coupling efficiencies for these two configurations are shown in the so-labeled columns of Fig. 9. As the figure shows, the basic coupling behavior between the modes is the same as has been observed in previous examples. The homogeneous mode coupling is essentially equal to the case of no defect. The coupling between modes $\mathrm{H}$ and $\mathrm{R}$ is approximately equivalent to that of a single sphere having a radius of $45 \mathrm{~nm}$ as shown in Fig. 9, and the coupling between other modes is negligible. As in previous results, the coupling due to defects placed closer to the probe base is slightly stronger than for those defects placed closer to the apex. We also consider a similar structure, which is a simple cylindrical tunnel through the midpoint of the metal layer, oriented perpendicular to the surface. The cylinder has a diameter of $20 \mathrm{~nm}$ and is assumed to consist of oxidized aluminum. The results are also shown in Fig. 9, with approximately the same behavior as the three-bubble tunnel.

Finally, we consider another type of defect, consisting of three identical spheres arranged in a line along the inner metal layer surface to simulate a localized region of surface roughness. We analyze the cases of this roughness defect being placed at the midpoint of the tapered section, as well as at the elbow of the probe. Each sphere has a diameter of $25 \mathrm{~nm}$, overlaps the adjacent spheres by $5 \mathrm{~nm}$, is tangent to the inner metal surface, and is taken to be composed of oxidized aluminum. As the results of Fig. 9 show, this defect also has a similar effect as in the previous cases in terms of induced coupling between the various modes.

Overall, this set of examples shows that a number of simple but more realistic defects also results in an induced coupling between modes $\mathrm{H}$ and $\mathrm{R}$. Comparing the results for a single $45 \mathrm{~nm}$ sphere at the midpoint of the taper, also shown in Fig. 9, with these five example defects, we find that the basic influence of all of the defects on the coupling of modes in the structure is approximately the same. Each of the fundamental modes is coupled to a like mode at the output with approximately the same efficiency as for a probe without defects. The introduction of the realistic defects induces a coupling between modes $\mathrm{H}$ and $\mathrm{R}$, with an exact strength that depends on the position and configuration of the defect. However, the qualitative behavior of this coupling is the same for all probe defect configurations studied.

\section{Discussion}

The various cases of multiple defects in the probe demonstrated in this section lead to an extrapolation of the effect of defects in a real probe-one having a large number of random, nonideal defects. As shown, when we consider the probes solely within the context of the three polarization eigenmodes, the effect of a single spherical defect is to introduce a coupling between one of the linear polarization modes and the radial/longitudinal polarization mode. We have also considered several cases of defects involving multiple spheres or other shapes. In a few specific cases (e.g., two identical spheres placed in symmetric positions on opposite sides of the probe), we have found destructive interference of the two scattering contributions. In contrast, for other configurations (e.g., two identical spheres in equivalent positions in orthogonal planes) the total coupling effect of the two spheres is simply the superposition of the effect of each individual sphere. From other configurations, we have observed a qualitatively additive behavior of the mode-coupling contribution of multiple defects. Finally, for differing defect shapes and configurations, although the magnitude of the coupling effect changes, the fundamental behavior is the same as for an effective spherical defect. Thus, for a large number of random defects, we expect the aggregate effect to be a coupling between each of the linear modes and the radial mode.

Of course, the quantitative strength of this coupling for a specific probe structure cannot be accurately determined from this approximate analysis. However, by comparing the results presented above, some important general conclusions can be made. In particular, we note that while the coupling coefficient from one input mode to a different output mode may, with a large defect or multiple defects, be many orders of magnitude higher than in the absence of defects, the coupling coefficient never approaches the same magnitude of the original input mode coupling to the matched output mode. This is primarily explained by the fact that the coupling is more or less bidirectional. For example, if the input mode is a linear polarization, the presence of defects will redirect a fraction of the input energy into the radial mode. However, the defects also introduce the reverse coupling from the radial mode to the original linear mode, and the strength of this secondary effect depends on the amount of energy in the radial mode (as well as many other factors, such as the precise defect characteristics and the field distribution inside the probe). In a simple analysis, the net coupling from linear to radial mode is essentially unidirectional when the strength of the linear mode is dominant, but gradually diminishes as the radial mode gains in strength, limiting the maximum ratio between the amplitudes of the two modes. Furthermore, in principle with a random defect array, we also expect coupling from the radial mode into the orthogonal linear mode. However, just as only a fraction of the energy in the input linear mode is coupled into the radial mode, we expect the absolute energy in the orthogonal linear mode to also be small. Consequently, with a very large number of random defects, we expect that the detailed effects of the specific shape, size, and position of a given single defect diminish in importance compared with the aggregate behavior of the large ensemble of defects. In other words, while the specific characteristics of a given real probe will still depend on the exact configuration of the defects, knowing that there are a large number of random defects allows us to draw the qualitative conclusion to expect a small but nontrivial amount of energy to be coupled from the input polarization mode to the other polarization modes.

Finally, it is important to realize that this approximate projection of the behavior of a large number of random defects is also limited to the regime where the defects constitute a perturbation on the behavior of the ideal probe model. For example, in the extreme case where defects are so prevalent that the metal coating becomes suffi- 
ciently perforated to yield an aperture, or even degraded to the extent that it can no longer be considered a layer, the optical properties will certainly be fundamentally different from this analysis. Further investigation, in simulation as well as in experiment, is needed to understand the behavior of probes in these different regimes, as well as to identify the demarcations between them. However, given the rapid advances in microfabrication and nanofabrication technologies of late, we believe it is reasonable to expect that probes with perturbative-rather than catastrophic - defects can be readily manufactured. While far from conclusive proof, this assumption is also consistent, at least qualitatively, with most experimental studies conducted so far. ${ }^{6,12,13}$

\section{CONCLUSIONS}

In this investigation, we have used rigorous electromagnetic modeling tools to determine the effect of simple defects in the metal layer of a SNOM probe model on the coupling of the polarization eigenmodes. We first performed a study of the effect of a single spherical defect of variable size on both the emitted optical near fields and the polarization mode coupling coefficients. Having found a correlation between these two properties, we were able to use the simpler mode coupling coefficient description in subsequent analysis, even though the emitted near field of the probe is actually the characteristic of interest. We then used this approach to examine a number of example defects, each consisting of one or a few simple inclusions in the probe metal-coating layer.

The most significant effect of a single defect was found to be the inducement of a coupling between a linearly polarized mode (with the electric field lying in the plane of the defect) and the radial/azimuthal polarization mode, which produces a highly localized emitted optical field at the apex of the probe. Of the defect configurations evaluated, however, not all were found to have the same effect on the mode coupling coefficients. The defect size most dramatically affected the coupling coefficients, varying over many orders of magnitude as the defect diameter ranged from 0 to $55 \mathrm{~nm}$ (with a $50 \mathrm{~nm}$ thick metalcoating layer). Also, the defect position was found to have a significant influence on the coupling coefficients, with defects placed close to the probe base inducing a stronger coupling than identical defects placed closer to the probe apex. In contrast, various defect configurations consisting of different shapes and clusters of small defects were found to yield much less variation in the resulting coupling coefficients. Although this ensemble of simplified probe models does not necessarily accurately represent the behavior of real, imperfect probes, a comparative analysis of these results provides useful information about the relative effect of several defect types and configurations on the induced coupling between polarization modes in the probe model, and consequently about the optical fields found inside and near the probe structure.

In addition, by comparing various configurations of multiple defects, we found that, although destructive interference or linear addition of the scattering contributions of the defects are observed for certain specific configurations, in most cases the contributions of the individual defects are weakly cumulative. Therefore, although the quantitative effect of each defect or set of defects depends on the specific configuration of the defect(s), we can make a reasonable qualitative prediction concerning the aggregate behavior of a large number of random defects. By extrapolating these results to the case of real probes having a large number of random defects, we expect a small but significant coupling of energy between the polarization modes in the probe.

For SNOM applications, conversion of a linearly polarized input mode into a radially/longitudinally polarized mode would be extremely beneficial due to the strong localization of this latter mode in the emitted near field of the probe. Although the amount of energy transferred in this manner would generally be small for a probe with random defects, the results of Section 3 show that, even for a single defect, in the highly confined hot-spot region near the apex of the probe, the emitted field attributable to the radial/longitudinal mode is dominant with respect to the input linearly polarized mode. Consequently, by optimizing the structure of the probe to improve the conversion efficiency or with polarization-based filtering of the collected light, it should be possible to obtain the benefits of the radially polarized mode using a linearly polarized input to the probe. The knowledge gained concerning the coupling of the polarization modes of the probe could facilitate this optimization of the probe structure, for example, by introducing specific intentional defects. In the future, more detailed study of these polarization modeconversion approaches, direct comparison of simulation and experimental results, and continued investigation of polarization effects in these structures are all necessary to better understand and optimize the optical characteristics of fully metal-coated microfabricated SNOM probes.

\section{ACKNOWLEDGMENTS}

The authors gratefully acknowledge the support of the Swiss National Science Foundation. The authors thank Ari T. Friberg, Department of Microelectronics and Information Technology, Royal Institute of Technology, Stockholm, Sweden, for his helpful comments and suggestions.

\section{REFERENCES}

1. R. Bachelot, P. Gleyzes, and A. C. Boccara, "Reflectionmode scanning near-field optical microscopy using an apertureless metallic tip,” Appl. Opt. 36, 2160-2170 (1997).

2. G. Schürmann, W. Noell, U. Staufer, and N. F. de Rooij, "Microfabrication of a combined AFM-SNOM sensor," Ultramicroscopy 82, 33-38 (2000).

3. R. Eckert, J. M. Freyland, H. Gersen, H. Heinzelmann, G. Schürmann, W. Noell, U. Staufer, and N. F. de Rooij, "Near-field fluorescence imaging with $32 \mathrm{~nm}$ resolution based on microfabricated cantilevered probes," Appl. Phys. Lett. 77, 3695-3697 (2000).

4. G. Schürmann, W. Noell, U. Staufer, N. F. de Rooij, R. Eckert, J. M. Freyland, and H. Heinzelmann, "Fabrication and characterization of a silicon cantilever probe with an integrated quartz-glass (fused-silica) tip for scanning nearfield optical microscopy," Appl. Opt. 40, 5040-5045 (2001).

5. J. L. Bohn, D. J. Nesbitt, and A. Gallagher, "Field enhancement in apertureless near-field scanning optical microscopy," J. Opt. Soc. Am. A 18, 2998-3006 (2001).

6. L. Aeschimann, T. Akiyama, U. Staufer, N. F. de Rooij, L. Thiery, R. Eckert, and H. Heinzelmann, "Characterization 
and fabrication of fully metal-coated scanning near-field optical microscopy SiO2 tips," J. Microsc. 209, 182-187 (2003).

7. R. Fikri, T. Grosges, and D. Barchiesi, "Apertureless scanning near-field optical microscopy: the need for probevibration modeling," Opt. Lett. 28, 2147-2149 (2003).

8. S. Aubert, A. Bruyant, S. Blaize, R. Bachelot, G. Lerondel, S. Hudlet, and P. Royer, "Analysis of the interferometric effect of the background light in apertureless scanning near-field optical microscopy," J. Opt. Soc. Am. B 20, 2117-2124 (2003).

9. L. J. Richter, C. E. Jordan, R. R. Cavanagh, G. W. Bryant, A. Liu, S. J. Stranick, C. D. Keating, and M. J. Natan, "Influence of secondary tip shape on illumination-mode near-field scanning optical microscopy images," J. Opt. Soc. Am. A 16, 1936-1946 (1999).
10. W.-X. Sun and Z.-X. Shen, "Optimizing the near field around silver tips," J. Opt. Soc. Am. A 20, 2254-2259 (2003).

11. L. Vaccaro, L. Aeschimann, U. Staufer, H. P. Herzig, and R. Dändliker, "Propagation of the electromagnetic field in fully coated near-field optical probes," Appl. Phys. Lett. 83, 584-586 (2003).

12. E. Descrovi, L. Vaccaro, W. Nakagawa, L. Aeschimann, U. Staufer, and H. P. Herzig, "Collection of transverse and longitudinal fields by means of apertureless nanoprobes with different metal coating characteristics," Appl. Phys. Lett. 85, 5340-5342 (2004).

13. E. Descrovi, L. Vaccaro, L. Aeschimann, W. Nakagawa, U. Staufer, and H. P. Herzig, "Optical properties of microfabricated fully metal-coated near-field probes in collection mode," J. Opt. Soc. Am. A 22, 1432-1441 (2005). 